\title{
Does Dose Modification Affect Efficacy of First-Line Pazopanib in Metastatic Renal Cell Carcinoma?
}

\author{
Paolo Grassi ${ }^{1}\left[\right.$ - Elena Verzoni ${ }^{1} \cdot$ Raffaele Ratta $^{1} \cdot$ Luca Porcu $^{2} \cdot$ Michele Prisciandaro $^{1}$. \\ Alessia Mennitto $^{1} \cdot$ Giuseppina Calareso $^{3}$ - Filippo de Braud ${ }^{1} \cdot$ Giuseppe Procopio $^{1}$
}

Published online: 11 August 2017

(c) The Author(s) 2017. This article is an open access publication

\begin{abstract}
Background Pazopanib is a standard treatment for metastatic renal cell carcinoma (mRCC), and $800 \mathrm{mg} / \mathrm{daily}$ is considered the optimal dose. However, some patients require dose modification because of toxicity. Whether a reduced dose of pazopanib is as effective as the standard dose in achieving clinical benefit remains unclear.

Objectives Our objective was to conduct a retrospective analysis to investigate the clinical effect of different therapeutic doses of first-line pazopanib in patients with mRCC.

Methods Consecutive patients with mRCC treated with first-line pazopanib between 2011 and 2016 at the Istituto Nazionale Tumori of Milan were retrospectively analysed for demographics, response, outcomes, and toxicity. Three patient groups were compared: group 1 received the standard dose of $800 \mathrm{mg} /$ day; group 2 started with $800 \mathrm{mg} /$ day and then reduced the dose to 400 or $600 \mathrm{mg} /$ day because of toxicity; and group 3 received a reduced starting dose of 400 or $600 \mathrm{mg} /$ day because they had an Eastern Cooperative Oncology Group (ECOG) performance status (PS) of 2 and/or comorbidities.

Results In total, 69 patients were evaluated: 34 in group 1,19 in group 2, and 16 in group 3. After a median
\end{abstract}

Paolo Grassi

paolo.grassi@istitutotumori.mi.it

1 Oncologia Medica 1, Fondazione IRCCS Istituto Nazionale Tumori, Via Venezian 1, 20133 Milan, Italy

2 IRCCS-Istituto di Ricerche Farmacologiche Mario Negri, Milan, Italy

3 Department of Radiology, Fondazione IRCCS Istituto Nazionale dei Tumori, Milan, Italy follow-up of 13.9 months (range 0.3-43.8), 27 (39.1\%) patients had progressive disease (PD) and three (4.3\%) patients had died. The incidence rate of PD or death per 100 person-months was 2.5 [95\% confidence interval (CI) 0.6-4.4; hazard ratio (HR) 1] in group 1 and 3.9 (95\% CI $0-14.3$; HR 1.43) in the combined group $(2+3)$. The discontinuation rate due to PD was $28 \%$ in group $1,42 \%$ in group 2, and $44 \%$ in group 3 . The objective response rate was 44,11 , and $19 \%$ in groups 1,2 , and 3, respectively.

Conclusions Our results may suggest that patients with mRCC receiving a lower dose of first-line pazopanib might not have a meaningful progression-free survival advantage compared with those receiving a standard dose. These data highlight that proper management of treatment-related side effects may lead to optimal drug exposure.

\section{Key Points}

Patients receiving first-line pazopanib for metastatic renal cell carcinoma (mRCC) often undergo dose modifications because of comorbidities or toxicity, but whether reduced-dose pazopanib is as effective as the standard dose in achieving a clinical benefit is unclear.

This study may suggest that patients with mRCC who receive a lower dose of first-line pazopanib might not have a meaningful progression-free survival advantage compared with those receiving a standard dose. 


\section{Introduction}

Agents that target angiogenesis through the vascular endothelial growth factor (VEGF) pathway are the backbone of therapy for metastatic renal cell carcinoma (mRCC) $[1,2]$. Variations in drug levels in patients treated with VEGF receptor (VEGFR) tyrosine kinase inhibitors (TKIs) represent a significant challenge in the treatment of patients with mRCC. Plasma exposure varies according to dose, and correlation is lacking between plasma levels and efficacy in individual patients [3, 4]. The pharmacokinetics of TKIs have demonstrated significant interindividual variability, which might contribute to the variable clinical response observed in patients receiving these drugs [5]. As a result, the variability in plasma exposure and clinical efficacy is reflected in the dosing recommendations for these TKIs. In addition, a number of factors affect the efficacy of a drug or treatment regimen in patients with cancer, including patient and tumor characteristics, tumor biology, and systemic exposure to the drug, which may vary because of schedule and/or dosing modifications due to toxicity [6]. Whether current dosing and schedules for TKIs are optimal in terms of achieving a maximum therapeutic response remains unclear, and a subset of patients will continue to have subtherapeutic drug levels such that progressive disease (PD) during VEGFR TKI therapy might not be a product of resistance to therapy but rather a result of underdosing. First-line pazopanib is one of the standard options for treatment-naïve mRCC [2], but it is frequently associated with adverse events (AEs) and dose reductions. The most common AEs related to pazopanib include fatigue, nausea, diarrhea, hypertension, anorexia, and hair depigmentation, as well as aspartate transaminase (AST) and alanine transaminase (ALT) elevations [2]. Liver abnormalities (3.8\%), diarrhea (2\%), and arterial thrombotic events $(2 \%)$ are the most common reasons for treatment discontinuation. Toxicity often leads to dose reductions or treatment discontinuation, which might improve tolerability; however, whether a reduced dose is comparable to a standard dose in achieving response or delaying disease progression is unclear. The goal of the present retrospective analysis was to investigate the clinical effect of different therapeutic doses of first-line pazopanib in patients with $\mathrm{mRCC}$.

\section{Materials and Methods}

\subsection{Patients and Treatment}

This was a single-center retrospective cohort study. Details of consecutive patients with mRCC treated with pazopanib as a first-line targeted therapy between 2011 and 2016 at the Istituto Nazionale Tumori of Milano (Italy), a comprehensive cancer center, were analyzed. Baseline demographics, including comorbidities and treatment duration, were evaluated. After reviewing the therapeutic schedule and dosage, investigators divided patients into three groups: patients who started treatment with the standard oral pazopanib $800 \mathrm{mg}$ daily and continued to receive the same dose throughout the treatment (group 1); patients who switched from oral pazopanib $800 \mathrm{mg}$ daily to oral pazopanib 600 or $400 \mathrm{mg}$ daily because of intolerable toxicity or patient refusal to continue treatment at the same dose (group 2); and frail subjects who started treatment with oral pazopanib $400 \mathrm{mg}$ or $600 \mathrm{mg}$ daily because of a low Eastern Cooperative Oncology Group (ECOG) performance status (PS) of 2 or comorbidities according to local treating clinical practice (group 3).

\subsection{Data Analysis}

The primary study outcome was progression-free survival (PFS), defined as the time from initiation of pazopanib therapy to the date of first documented disease progression, loss to follow-up, or death due to any cause, whichever occurred first. Secondary study outcomes included treatment duration, discontinuation rate, and treatment response. Treatment response was assessed using the RECIST (Response Evaluation Criteria in Solid Tumors) version 1.1 criteria [7], whereas requirements for dose reduction and assessment of toxicity were evaluated according to CTCAE (Common Terminology Criteria for Adverse Events) version 4.0 [8].

\subsection{Statistical Analyses}

The PFS incidence rate was calculated as the ratio between the number of PFS events and the sum of total PFS time contributed by all patients. Because of the treatment shift to a reduced dose from group 1 to group 2, survival functions were estimated using the Simon-Makuch method. The Cox regression model was used to detect the statistical association and to estimate the hazard ratio (HR). Median followup time was estimated using the reverse Kaplan-Meier method. The objective response rate (ORR) was calculated as the percentage of complete and partial treatment response. Baseline covariate distributions and treatment characteristics were summarized using descriptive statistics (median and range for continuous variables; absolute and percentage frequencies for categorical variables). All statistical tests were two-sided. Given the descriptive nature of the study, hypothesis testing was applied qualitatively (i.e., no threshold for statistical significance was defined). Statistical analysis was performed using SAS version 9.2 
(SAS Institute Inc., Cary, NC, USA). The Kaplan-Meier curves were plotted using STATA software version 12.1 (StataCorp, College Station, TX, USA).

\section{Results}

From June 2011 to September 2016, a total of 69 patients started treatment with first-line pazopanib; 34 (49\%), 19 $(28 \%)$, and 16 subjects (23\%) were evaluated in groups 1 , 2 , and 3 , respectively. The median age at diagnosis was 62 years, and $64 \%$ were male. Overall, $13 \%$ and $87 \%$ of patients were classified as Heng 'good' and 'intermediate' risk, respectively. Patients' baseline characteristics are reported in Table 1. Cardiovascular comorbidities at baseline were observed in ten (29\%), six (32\%), and 14 $(88 \%)$ patients in groups 1,2 , and 3 , respectively (Table 1 ).

\subsection{Treatment and Adverse Event Profile}

After starting oral pazopanib treatment at the full dose (800 mg daily), 16 patients (84\%) reduced the dose to $400 \mathrm{mg}$ daily, and three (16\%) reduced the dose to $600 \mathrm{mg}$ daily (overall, 19 patients who were defined as group 2). The most frequent reasons for reducing the dose were grade 2 fatigue $(n=2 ; 11 \%)$, mucositis $(n=1 ; 5 \%)$, diarrhea $(n=1 ; 5 \%)$, nausea $(n=3 ; 16 \%)$, neutropenia $(n=1 ; 5 \%)$, mucositis $(n=1 ; 5 \%)$, hypertransaminasemia $(n=3 ; 16 \%)$, and hypertension $(n=3 ; 16 \%)$ and grade 3 hypertension $(n=7 ; 37 \%)$, hypertransaminasemia $(n=4 ; 21 \%)$, and fatigue $(n=8 ; 42 \%)$. In total, $34(56 \%)$ patients who started on pazopanib $800 \mathrm{mg}$ daily maintained the full dose of pazopanib. The most frequent toxicities reported among these patients were grade 1 diarrhea $(n=5 ; 33 \%)$, fatigue $(n=7 ; 47 \%)$, hypertension $(n=1$; $7 \%)$, mucositis $(n=1 ; 7 \%)$, and skin rash $(n=1 ; 7 \%)$ and grade 2 diarrhea $(n=2 ; 13 \%)$, nausea $(n=1 ; 7 \%)$, vomiting $(n=1 ; 7 \%)$, fatigue $(n=2 ; 13 \%)$, and hypertension $(n=1 ; 7 \%)$. In group 3 , a total of $12(75 \%)$ subjects started treatment at a reduced dose of $400 \mathrm{mg}$ daily, and four $(25 \%)$ started at $600 \mathrm{mg}$ daily. The most frequent reason for starting treatment at a reduced dose was cardiovascular comorbidity, including uncontrolled hypertension in seven patients $(44 \%)$, previous acute myocardial infarction in three patients (19\%), previous transient ischemic attack in two patients (12.5\%), arrhythmias in three patients (19\%), and Felty's syndrome in one case, and three of these patients presented with an ECOG PS of 2 (Table 1). The incidence of some grade 3 toxicities commonly associated with pazopanib were fatigue $(20 \%$ in group 1 vs. $6 \%$ in group $2+3)$, hypertension $(13 \%$ in group 1 vs. $0 \%$ in group $2+3$ ), and liver function test failure (6\% in group 1 vs. $0 \%$ in group $2+3$ ) (Table 2 ).

\subsection{Treatment Duration and Survival}

After a median follow-up of 13.9 months (range 0.3-43.8), $27(39.1 \%)$ patients had PD and three (4\%) patients had died (total PFS events 30). At the date of last follow-up, 17 $(50 \%)$, seven (37\%), and six (38\%) patients were alive and receiving treatment in groups 1,2 , and 3 , respectively, whereas $17(50 \%), 12(63 \%)$, and ten $(62 \%)$ patients had discontinued treatment because of PD or toxicity or were lost to follow-up. Patients had been taking pazopanib for a median of 9.6 months (range 0.3-43.8) in group 1, for 13.6 months (range 0.1-29.5) in group 2, and for 18.3 months in group 3. The PFS incidence rate per 100 person-months was $2.5[95 \%$ confidence interval (CI) 0.6-4.4; HR 1] in group 1 and 3.9 [95\% CI 0.68-2.98; HR 1.43 (95\% CI 0.68-2.98)] in group $2+3$ (Table 3). Rates of discontinuation due to PD were $28 \%$ (15 patients) in group 1, 42\% (eight patients) in group 2, and $44 \%$ (seven patients) in group 3 . The ORR was 44,11 , and $19 \%$ in groups 1,2 , and 3 , respectively. When dose-reduction groups 2 and 3 were considered together and defined as a group $(2+3)$, the PFS incidence rates were as summarized in Table 3. Median PFS was not reached in group 1 and was 17.9 months (95\% CI 7.6-44.8) for group $2+3$ (Figs. 1, 2). Before a PFS event, patients had been taking pazopanib for a median of 9.6 months (range 0.3-43.8) in group 1, for 13.6 months (range 0.1-29.5) in group 2, and for 18.3 months (range 2.1-31.4) in group 3. At the univariate analysis, the dose-reduction group $(2+3)$ was associated with less favorable survival (HR $1.43 ; 95 \%$ CI $0.68-2.98 ; p=0.343$ ) than was group 1 . The multivariate analysis confirmed that the worse outcome was associated with dose reduction (HR 1.44; 95\% CI 0.69-3.00) for the dose-reduction group $(2+3)$, whereas the Heng prognostic score discriminated between a 'good' and an 'intermediate' score (HR 0.66; 95\% CI 0.20-2.17; $p=0.501$ ) in the overall population analysed. In group 1 , three patients experienced complete response (CR), 12 patients experienced partial response (PR), and 11 patients experienced stable disease (SD), with an ORR (CR + PR) of $44 \%$. In group 2, no CRs were reported, whereas two and seven patients achieved PR and SD, respectively, with an ORR of $11 \%$. In group 3, no CRs were seen, whereas three patients reported a PR and four patients reported SD, with an ORR of 19 (Table 4).

\section{Discussion}

Pazopanib is a recommended first-line option for the treatment of mRCC $[9,10]$. Retrospective data have shown that an increased median PFS and tumor shrinkage appear in patients with higher plasma trough drug levels $\left(C_{\mathrm{min}}\right)$ 
Table 1 Patient characteristics

\begin{tabular}{|c|c|c|c|c|}
\hline Characteristic & Full dose $(N=34)$ & Dose reduction $(N=19)$ & Starting dose reduction $(N=16)$ & Total patients $(N=69)$ \\
\hline \multicolumn{5}{|l|}{ Sex } \\
\hline Male & $27(79)$ & $6(32)$ & $10(63)$ & $43(62)$ \\
\hline Female & $7(21)$ & $13(68)$ & $6(37)$ & $26(38)$ \\
\hline \multicolumn{5}{|l|}{ Median age (years) } \\
\hline \multicolumn{5}{|c|}{ ECOG performance status } \\
\hline 0 & $27(79)$ & $18(95)$ & $6(38)$ & $51(74)$ \\
\hline 1 & $6(18)$ & $1(5)$ & $7(44)$ & $14(20)$ \\
\hline 2 & $1(3)$ & - & $3(19)$ & $4(6)$ \\
\hline \multicolumn{5}{|l|}{ Comorbidities } \\
\hline Cardiovascular & $10(29)$ & $6(32)$ & $14(88)$ & $30(43)$ \\
\hline Diabetes mellitus & $4(12)$ & $2(11)$ & $4(25)$ & $10(14)$ \\
\hline Other & $2(6)$ & $2(11)$ & $2(13)$ & $6(9)$ \\
\hline \multicolumn{5}{|l|}{ Histology } \\
\hline Clear cell & $34(100)$ & $19(100)$ & $15(94)$ & $68(99)$ \\
\hline Non-clear cell & - & - & $1(6)$ & $1(1)$ \\
\hline \multicolumn{5}{|l|}{ Nephrectomy } \\
\hline Yes & $32(94)$ & $19(100)$ & $16(100)$ & $67(97)$ \\
\hline No & $2(6)$ & - & - & $2(3)$ \\
\hline \multicolumn{5}{|l|}{ Heng score } \\
\hline Good & $6(18)$ & $2(811)$ & $2(13)$ & $10(14)$ \\
\hline Intermediate & 27 (79) & $17(89)$ & $14(88)$ & $58(84)$ \\
\hline Poor & $1(3)$ & - & - & $1(1)$ \\
\hline \multicolumn{5}{|l|}{ Metastatic sites $(N)$} \\
\hline 1 & $14(41)$ & $8\left(44^{\mathrm{a}}\right)$ & $7(44)$ & $29(42)$ \\
\hline 2 & $19(56)$ & $7\left(39^{\mathrm{a}}\right)$ & $6(38)$ & $32(46)$ \\
\hline $3-6$ & $2(6)$ & $3\left(17^{\mathrm{a}}\right)$ & $3(19)$ & $8(12)$ \\
\hline \multicolumn{5}{|l|}{ Metastatic sites } \\
\hline Lymph node & $14(41)$ & $2\left(11^{\mathrm{a}}\right)$ & $6(38)$ & $22(32)$ \\
\hline Bone & $9(26)$ & $3\left(17^{\mathrm{a}}\right)$ & $2(13)$ & $14(20)$ \\
\hline Lung & $3(9)$ & $7\left(39^{\mathrm{a}}\right)$ & $2(13)$ & $12(17)$ \\
\hline Pancreas & $3(9)$ & $7\left(39^{\mathrm{a}}\right)$ & $2(13)$ & $12(17)$ \\
\hline Contralateral kidney & $1(3)$ & $2\left(11^{\mathrm{a}}\right)$ & $2(13)$ & $5(7)$ \\
\hline Liver & $2(6)$ & $3\left(17^{\mathrm{a}}\right)$ & $2(13)$ & $7(10)$ \\
\hline Other $^{\mathrm{b}}$ & $7(21)$ & $1(5)$ & $3(19)$ & $11(16)$ \\
\hline
\end{tabular}

Data are presented as $N(\%)$ unless otherwise indicated

ECOG Eastern Cooperative Oncology Group, PS performance status

${ }^{a}$ Sites of disease missing for one patient

b Pleura, brain, skin, adrenal gland

[11]. Therefore, patients with a low $C_{\min }$ might benefit from pharmacokinetically guided individualized dosing. Dose reductions to pazopanib $400 \mathrm{mg}$ occurred in $30 \%$ of patients with RCC in the phase II study [12]. The most common AEs leading to dose interruptions or reductions were diarrhea, hypertension, and increased ALT/AST levels [12]. Results suggest that the frequency of these common AEs could be reduced by decreasing systemic exposure to pazopanib through dose reductions or interruptions. However, interpatient heterogeneity in the metabolism and/or absorption of pazopanib can lead to differences in systemic exposure. Nevertheless, in clinical practice, both comorbidities and AEs might limit the pazopanib dosage and affect therapeutic response. To investigate the greatest benefit, we analyzed the relationship between different pazopanib doses and efficacy. In our 
Table 2 Pazopanib administration and adverse events profile

\begin{tabular}{|c|c|c|c|c|}
\hline Characteristics & Group 1 & Group 2 & Group 3 & Total \\
\hline \multicolumn{5}{|c|}{ Pazopanib starting dose (mg/daily) } \\
\hline 800 & $53(77)$ & $19(36)$ & - & $53(77)$ \\
\hline 600 & - & - & $4(25)$ & $4(6)$ \\
\hline 400 & - & - & $12(75)$ & $12(17)$ \\
\hline \multicolumn{5}{|c|}{ Dose reduction (mg/daily) } \\
\hline Yes & $19(36)$ & $19(36)$ & $16(23)$ & $35(51)$ \\
\hline 600 & & $3(6)$ & - & $3(4)$ \\
\hline 400 & & $16(30)$ & - & \\
\hline No & $34(64)$ & - & - & $34(49)$ \\
\hline \multicolumn{5}{|l|}{ Fatigue } \\
\hline Grade 1 and 2 & $9(27)$ & $2(11)$ & $9(56)$ & $20(29)$ \\
\hline Grade 3 & $1(3)$ & $1(5)$ & $1(6)$ & $3(4)$ \\
\hline \multicolumn{5}{|l|}{ Hypertension } \\
\hline Grade 1 and 2 & $4(8)$ & $3(16)$ & $2(12)$ & $9(13)$ \\
\hline Grade 3 & 2 & - & - & $2(3)$ \\
\hline \multicolumn{5}{|l|}{ Mucositis } \\
\hline Grade 1 and 2 & $1(3)$ & $1(5)$ & - & $2(3)$ \\
\hline Grade 3 & - & - & - & - \\
\hline \multicolumn{5}{|l|}{ Diarrhea } \\
\hline Grade 1 and 2 & $7(21)$ & $2(11)$ & - & $9(13)$ \\
\hline Grade 3 & - & $3(16)$ & - & $3(4)$ \\
\hline \multicolumn{5}{|l|}{ Nausea } \\
\hline Grade 1 and 2 & $1(3)$ & $3(16)$ & $7(44)$ & $11(16)$ \\
\hline Grade 3 & $1(3)$ & - & - & $1(1)$ \\
\hline \multicolumn{5}{|l|}{ Liver toxicity } \\
\hline Grade 1 and 2 & $1(3)$ & $3(16)$ & $2(12)$ & $6(9)$ \\
\hline Grade 3 & $1(3)$ & - & - & $1(1)$ \\
\hline \multicolumn{5}{|l|}{ Skin rash } \\
\hline Grade 1 and 2 & $1(3)$ & - & - & $1(1)$ \\
\hline Grade 3 & - & - & - & - \\
\hline
\end{tabular}

Data are presented as point estimate (95\% confidence interval) unless otherwise indicated

$N A$ not applicable

${ }^{\text {a }}$ Incidence rate is presented as events per 100 person-months

study, the majority of patients started on the standard dose of pazopanib $800 \mathrm{mg}$ daily; however, $19(36 \%)$ of these patients required a dose reduction, and $16(23 \%)$ patients started pazopanib on a reduced dose because of comorbidities. On the other hand, $14(41 \%)$, nine $(47.4 \%)$, and nine $(56.3 \%)$ patients in groups 1,2 , and 3, respectively, discontinued treatment because of PD or toxicity. The most common AEs observed in our study were fatigue (33.3\%), nausea $(17.4 \%)$, diarrhea (16\%), and hypertension (13\%), which is consistent with data in the literature [2]. Treatment duration and toxicity data from real-world experience with sunitinib and sorafenib show that, overall, about half of patients require a dose reduction of sunitinib [13] and sorafenib $[14,15]$ because of toxicity; however, no similar
Table 3 Progression-free survival incidence rate and hazard ratio for groups 1 and $(2+3)$

\begin{tabular}{llll}
\hline Group & \multicolumn{1}{c}{ Incidence rate $^{\mathrm{a}}$} & Hazard ratio & $p$ value \\
\hline 1 & $2.5(0.6-4.4)$ & $1(\mathrm{NA})$ & 0.343 \\
$(2+3)$ & $3.9(0-14.3)$ & $1.43(0.68-2.98)$ & \\
Multivariate analysis & & \\
Group & 1 (NA) & 0.334 \\
1 & $1.44(0.69-3.00)$ & \\
$(2+3)$ & & \\
Heng score & $1(\mathrm{NA})$ & 0.488 \\
Poor-intermediate & $0.66(0.20-2.17)$ & \\
Good & & \\
\hline
\end{tabular}

Data are presented as $n(\%)$ 


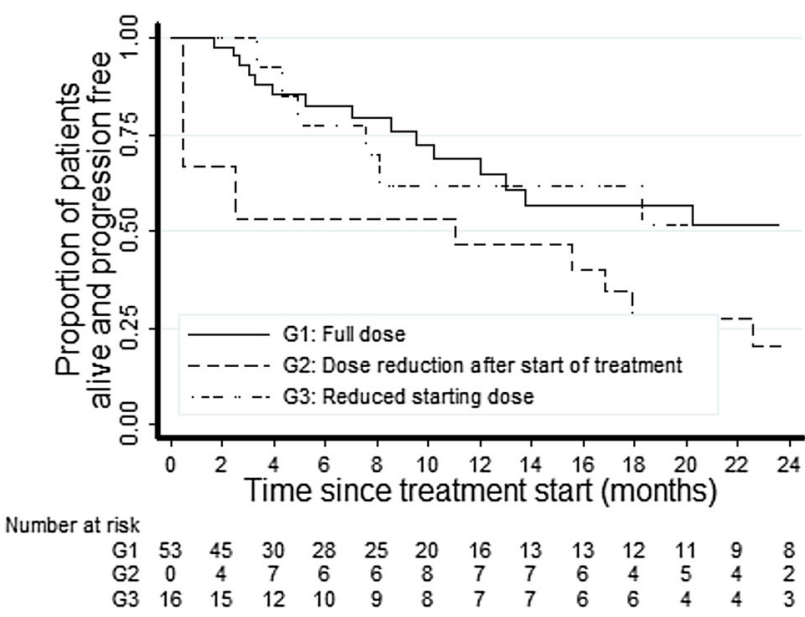

Fig. 1 Progression-free survival curves for groups 1 (G1), 2 (G2), and $3(\mathrm{G} 3)$

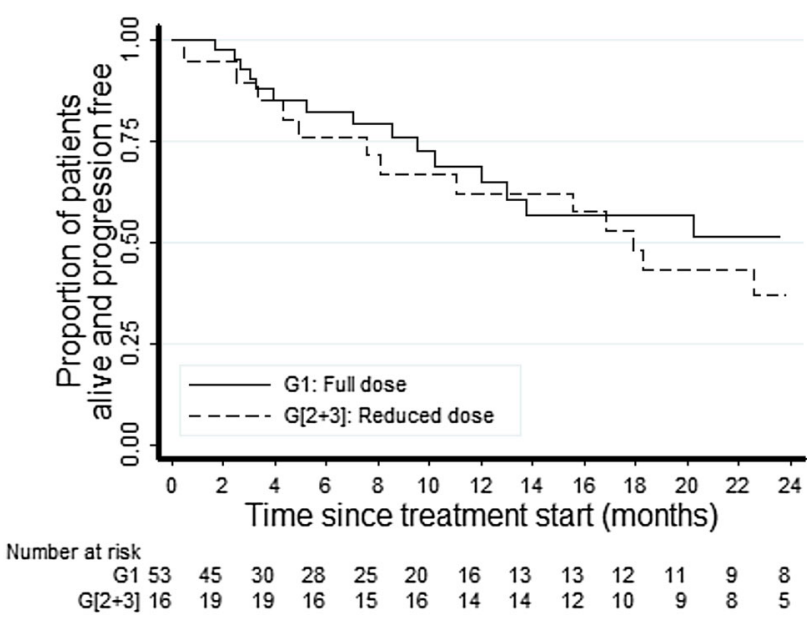

Fig. 2 Progression-free survival curves for group 1 (G1) and groups 2 and 3 combined [G $(2+3)]$ data are yet available for pazopanib. The disease control rate (DCR) in our report $(n=20 ; 29 \%)$ is slightly lower than that reported in the literature, but it raised significantly when considering the DCR reported in group 1 (44\%). The median PFS (not reported for group 1 and 17.9 months for group $2+3$ ) was higher than that reported in other studies $[9,10]$, but this might reflect the small sample considered in the dose reduction cohort as well as the clinical management of side effects leading to longer treatment exposure. Moreover, only subjects with clear cell histology and good and intermediate prognosis treated with first-line pazopanib were included in the study as per clinical practice in Italy. At both univariate and multivariate analysis, dose reduction was associated with worse outcomes. These results should be interpreted with great caution because of methodological limits. Moreover, sampling variability was high because of the low number of PFS events collected. Imbalance of other confounders not considered in the multivariate regression model could heavily bias effect estimates. Different PFS assessments between groups and time-varying PFS incidence rates could also bias effect estimates. The lack of a preplanned hypothesis to test, the retrospective nature of the data, the single institution experience, and the small sample size must also be taken into consideration in terms of the reliability and generalizability of this study. In light of this variability and the relationship between systemic exposure and clinical benefit and toxicities, dose adjustments based on plasma concentrations may provide a method to optimize pazopanib therapy by maintaining therapeutically effective blood levels and minimizing AEs. To the best of our knowledge, this is the first study to report outcomes for patients treated with reduced-dose first-line pazopanib and may represent a useful addition to the growing literature on TKIs in $\mathrm{mRCC}$.

Table 4 Response rates

\begin{tabular}{lllc}
\hline Characteristic & Group 1 & Group 2 & Group 3 \\
\hline Response rate & & & Total \\
CR & $3(8.8)$ & - & - \\
PR & $12(35.3)$ & $2(10.5)$ & $3(18.8)$ \\
SD & $11(32.3)$ & $7(36.8)$ & $4(25)$ \\
PD & $8(23.5)$ & $2(10.5)$ & $3(18.8)$ \\
NA & - & $1(5.2)$ & - \\
Present status & & $13(19)$ \\
Alive, on treatment & $17(50)$ & $7(37)$ & $6(38)$ \\
Drug discontinued due to death/progressive disease/toxicity/drug holiday & $17(50)$ & $12(63)$ & $10(62)$ \\
\hline
\end{tabular}

Data are presented as $n(\%)$

$C R$ complete response, $N A$ not available, $P D$ progressive disease, $P R$ partial response, $S D$ stable disease 


\section{Conclusions}

Our data may suggest that patients with $\mathrm{mRCC}$ receiving a lower dose of first-line pazopanib might not have a meaningful PFS advantage compared with those receiving a standard dose. These results highlight that the optimal management of treatment-related side effects may eventually lead to proper drug exposure.

\section{Compliance with Ethical Standards}

Conflict of interest Paolo Grassi, Elena Verzoni, Raffaele Ratta, Luca Porcu, Michele Prisciandaro, Alessia Mennitto, Giuseppina Calareso, Filippo de Braud, and Giuseppe Procopio have no conflicts of interest.

Funding No sources of funding were used to conduct this study or prepare this manuscript.

Open Access This article is distributed under the terms of the Creative Commons Attribution-NonCommercial 4.0 International License (http://creativecommons.org/licenses/by-nc/4.0/), which permits any noncommercial use, distribution, and reproduction in any medium, provided you give appropriate credit to the original author(s) and the source, provide a link to the Creative Commons license, and indicate if changes were made.

\section{References}

1. Motzer RJ, Hutson TE, Tomczak P, Michaelson MD, Bukowski $\mathrm{RM}$, Rixe $\mathrm{O}$, et al. Sunitinib versus interferon alfa in metastatic renal-cell carcinoma. N Engl J Med. 2007;356(2):115-24.

2. Sternberg CN, Davis ID, Mardiak J, Szczylik C, Lee E, Wagstaff $\mathrm{J}$, et al. Pazopanib in locally advanced or metastatic renal cell carcinoma: results of a randomized phase III trial. J Clin Oncol. 2010;28(6):1061-8.

3. Rini BI, Melichar B, Fishman MN, Oya M, Pithavala YK, Chen $\mathrm{Y}$, et al. Axitinib dose titration: analyses of exposure, blood pressure and clinical response from a randomized phase II study in metastatic renal cell carcinoma. Ann Oncol. 2015;26(7): 1372-7.

4. Rini BI, Melichar B, Ueda T, Grünwald V, Fishman MN, Arranz JA, et al. Axitinib with or without dose titration for first-line metastatic renal-cell carcinoma: a randomised double-blind phase 2 trial. Lancet Oncol. 2013;14(12):1233-42.

5. Klümpen HJ, Samer CF, Mathijssen RH, Schellens JH, Gurney H. Moving towards dose individualization of tyrosine kinase inhibitors. Cancer Treat Rev. 2011;37(4):251-60.

6. Lee JL, Kim MK, Park I, Ahn JH, Lee DH, Ryoo HM, et al. RandomizEd phase II trial of Sunitinib four weeks on and two weeks off versus two weeks on and one week off in metastatic clear-cell type REnal cell carcinoma: RESTORE trial. Ann Oncol. 2015;26(11):2300-5.

7. Eisenhauer EA, Therasse P, Bogaerts J, Schwartz LH, Sargent D, Ford R, et al. New response evaluation criteria in solid tumours: revised RECIST guideline (version 1.1). Eur J Cancer. 2009;45:228-47.

8. US Department of Health and Human Services; National Institutes of Health; National Cancer Institute. Common Terminology Criteria for Adverse Events (CTCAE). Version 4.0. NCI, NIH, DHHS. 2009. https://evs.nci.nih.gov/ftp1/CTCAE/CTCAE_4.03_ 2010-06-14_QuickReference_5x7.pdf

9. Motzer RJ, Hutson TE, Cella D, Reeves J, Hawkins R, Guo J, et al. Pazopanib versus sunitinib in metastatic renal-cell carcinoma. N Engl J Med. 2013;369(8):722-31.

10. Escudier B, Porta C, Bono P, Powles T, Eisen T, Sternberg CN, et al. Randomized, controlled, double-blind, cross-over trial assessing treatment preference for pazopanib versus sunitinib in patients with metastatic renal cell carcinoma: PISCES Study. J Clin Oncol. 2014;32(14):1412-8.

11. Suttle AB, Ball HA, Molimard M, Hutson TE, Carpenter C, Rajagopalan D, et al. Relationships between pazopanib exposure and clinical safety and efficacy in patients with advanced renal cell carcinoma. Br J Cancer. 2014;111(10):1909-16.

12. Hutson TE, Davis ID, Machiels JP, De Souza PL, Rottey S, Hong $\mathrm{BF}$, et al. Efficacy and safety of pazopanib in patients with metastatic renal cell carcinoma. J Clin Oncol. 2010;28(3): 475-80.

13. Gore ME, Szczylik C, Porta C, Bracarda S, Bjarnason GA, Oudard S, et al. Final results from the large sunitinib global expanded access trial in metastatic renal cell carcinoma. Br J Cancer. 2015;113(1):12-9.

14. Jäger D, Ma JH, Mardiak J, Ye DW, Korbenfeld E, Zemanova M, et al. Sorafenib treatment of advanced renal cell carcinoma patients in daily practice: the large international PREDICT study. Clin Genitourin Cancer. 2015;13(2):156-64.e1.

15. Procopio G, Bellmunt J, Dutcher J, Bracarda S, Knox J, Brueckner A, et al. Sorafenib tolerability in elderly patients with advanced renal cell carcinoma: results from a large pooled analysis. Br J Cancer. 2013;108(2):311-8. 